\title{
Immunoperoxidase study of the secretory immunoglobulin system and lysozyme in normal and diseased gastric mucosa
}

\author{
P ISAACSON* \\ From the Department of Pathology, Southampton University Medical School, Southampton
}

SUMMARY Using an immunoperoxidase technique the distribution of secretory component, IgA, and lysozyme has been investigated in normal, inflamed, dysplastic, and carcinomatous gastric mucosa. Apart from pyloric glands which contain lysozyme, normal gastric mucosa stains negatively for all three antigens. In gastritis mucous neck cells appear to adapt by synthesising secretory component and lysozyme and transporting IgA. Intense staining for the three antigens is seen in dysplastic gastric epithelium and in well-differentiated intestinal type carcinomas. With progressive de-differentiation the tumours lose the ability to synthesise secretory component and lysozyme. Carcinomas of the diffuse type stain positively for secretory component and lysozyme and individual cells appear to take up $\operatorname{IgA}$ even in the absence of surrounding $\operatorname{IgA}$ containing plasma cells. These functional properties are retained in lymph node metastases. It is suggested that secretory component synthesising malignant cells might take up circulating dimeric $\operatorname{IgA}$ and that this could be a reflection of an important physiological mechanism.

The secretory immunoglobulin (Ig) system comprises a complex series of events whereby dimeric IgA secreted by mucosal plasma cells is attached to secretory component, synthesised by mucosal epithelial cells, and then transported as the secretory component IgA complex through the epithelial cells to the mucosal surface. ${ }^{1}$ The different components of the system can be demonstrated by immunohistochemical means and in the small and large intestine the use of immunofluorescence and immunoperoxidase techniques has permitted detailed correlation of the functional aspects of secretory immunity with inflammatory and neoplastic disorders. ${ }^{2-6}$ Little attention has, however, been given to secretory immunity in the normal or diseased stomach. Like the components of the secretory Ig system immunohistochemical techniques can be used to demonstrate lysozyme in the gastrointestinal tract where it is found in small intestinal Paneth cells and some goblet cells $s^{5}$ and in the duodenal Brunner's glands. ${ }^{8}$ Lysozyme has

\footnotetext{
* Address for correspondence: Dr P Isaacson, Department of Pathology, Level E, South Laboratory Block, General Hospital, Southampton SO9 4XY. England.

Received for publication 20 November 1981
}

recently also been shown in gastric glands. ${ }^{9}$ The aims of the present study were to establish the distribution of the various components of the secretory Ig system and of lysozyme in the normal stomach and to observe the changes that occur in gastritis, gastric dysplasia, and gastric carcinoma.

\section{Methods}

The material studied consisted of formalin-fixed paraffin embedded gastric mucosa from blocks taken routinely from a total of 40 gastrectomy specimens which were considered to have been optimally fixed and to be suitable for immunoperoxidase stains. Suitable blocks of normal gastric body mucosa were obtained from three stomachs resected for duodenal ulcer. Entirely normal antral mucosa - that is, with no superficial mucosal inflammatory infiltrate - was not present in any of the specimens but blocks showing minimal inflammation were obtained from three of the 27 cases of carcinoma in which the tumour was situated in the cardia. Blocks showing gastritis of varying severity were selected from 10 cases of gastric ulcer and from 14 of the 27 cases of carcinoma. Sections from all 27 tumours were studied together with sections of gastric mucosal dysplasia present in five of the 27 
cases of carcinoma. Sections of lymph nodes containing metastatic tumour were available in 11 of the 27 cases of carcinoma and were also studied.

All sections were stained with haematoxylin and eosin and by the PAS method for mucin. Adjacent sections were stained by the PAP immunoperoxidase technique, following trypsin digestion, for secretory component, IgA ( $\alpha$-chain), and lysozyme. ${ }^{10}$ The rabbit antisera to $\operatorname{IgA}$ and secretory component were purchased from Behringwerke Hoechst UK Ltd, Hounslow, England, and the antiserum to lysozyme from Dako-immunoglobulins a/s Copenhagen, Denmark. Specificity controls for the antisera to $\operatorname{IgA}$ and lysozyme have been previously described. ${ }^{11}$ The positive staining reaction of rabbit anti-secretory component used at a dilution of $1 / 1000$ could be blocked by prior application of goat anti-secretory component (1/100) obtained from Nordic Immunological Reagents, Maidenhead, England. Additional controls consisted of the substitution of non-immune rabbit antiserum in place of specific antisera in which case no staining reaction was obtained.

\section{Results}

These are partially summarised in the Table.

In preparations stained for lysozyme there was always a considerable background of positively stained neutrophils, eosinophils, and macrophages. Epithelial cells staining for secretory component always stained with similar intensity for IgA regardless of the number of surrounding IgA containing plasma cells.

Table Immunohistochemical staining of gastric mucosa

\begin{tabular}{|c|c|c|c|}
\hline & $S C$ & $\operatorname{Ig} A$ & Lys \\
\hline \multicolumn{4}{|c|}{ Normal mucosa } \\
\hline Fov & - & - & - \\
\hline $\mathrm{MN}$ & $-*$ & $-^{*}$ & $-^{*}$ \\
\hline BG & - & - & - \\
\hline Pyl & - & - & + \\
\hline \multicolumn{4}{|l|}{ Gastritis } \\
\hline Fov & - & - & - \\
\hline $\mathbf{M N}$ & + & + & + \\
\hline BG & - & - & - \\
\hline Pyl & - & - & + \\
\hline IM & + & + & $+t$ \\
\hline \multicolumn{4}{|l|}{ Carcinoma } \\
\hline Intestinal & $\pm \ddagger$ & $\pm \ddagger$ & $\pm \ddagger$ \\
\hline Diffuse & + & + & + \\
\hline
\end{tabular}

Fov $=$ foveolae, $\mathrm{MN}=$ mucus neck, $\mathrm{BG}=$ gastric body glands, $\mathrm{Pyl}=$ pyloric glands, $\mathrm{IM}=$ intestinal metaplasia.

* Weakly positive in antrum - see text.

$\dagger$ Paneth cells and isolated goblet cells.

$\ddagger$ Positive staining in well-differentiated tumours fading to negative with progressive dedifferentiation.
NORMAL MUCOSA

In normal gastric body mucosa no secretory component was detected (Fig. 1) while only scattered plasma cells stained for IgA. Staining for lysozyme was likewise negative. In the strict sense of the term no 'normal' antral mucosa was available for study. A slight degree of chronic superficial gastritis was always seen in sections of antrum from gastrectomy specimens and can perhaps be considered a normal finding in the adult stomach. In antral mucosa the foveolar mucous cells stained negatively for secretory component but became positive in the isthmus or mucous neck region with concentration of staining along the apical border of the glands. Plasma cells were more numerous in the lamina propria, most staining positively for $\operatorname{IgA}$ which otherwise followed secretory component in its distribution and staining intensity. Pyloric glands almost always stained negatively for secretory component but occasionally faint positive staining was seen. These glands were strongly positive for lysozyme which was also present in mucous neck cells of the isthmus.

\section{GASTRITIS}

In gastritis an accumulation of $\operatorname{IgA}$ positive plasma cells in the lamina propria was accompanied by positive staining of the foveolar isthmus for secretory component, $\operatorname{IgA}$, and lysozyme in gastric body mucosa (Fig. 2) and intensification of staining in the same region in the antrum. Foveolar mucous cells, however, continued to stain negatively for all three antigens. Foci of intestinal metaplasia were highlighted by their strong secretory component staining with the pattern of staining similar to that of the crypts of small intestinal villi. Positive staining for lysozyme was seen in Paneth cells and isolated goblet cells (Fig. 3).

Regenerating mucosal epithelium at the edges of peptic ulcers stained variably positive for all three antigens but never as intensely as intestinal metaplasia or foci of dysplasia (see below).

\section{DYSPLASIA}

The diagnosis of dysplasia was based on the criteria of Morson et $\mathrm{al}^{12}$ and in each of the five cases the changes gradually merged into those characteristic of carcinoma either invading the mucosa or beyond. The dysplastic glands were composed of atypical cells containing large hyperchromatic nuclei with a tendency to stratification and loss of polarity. There was marked reduction in mucin secretion compared with the surrounding mucosa and this was well shown by the PAS stain. The dysplastic glands showed irregular budding and papillary infolding with a degree of crowding falling short of frank 


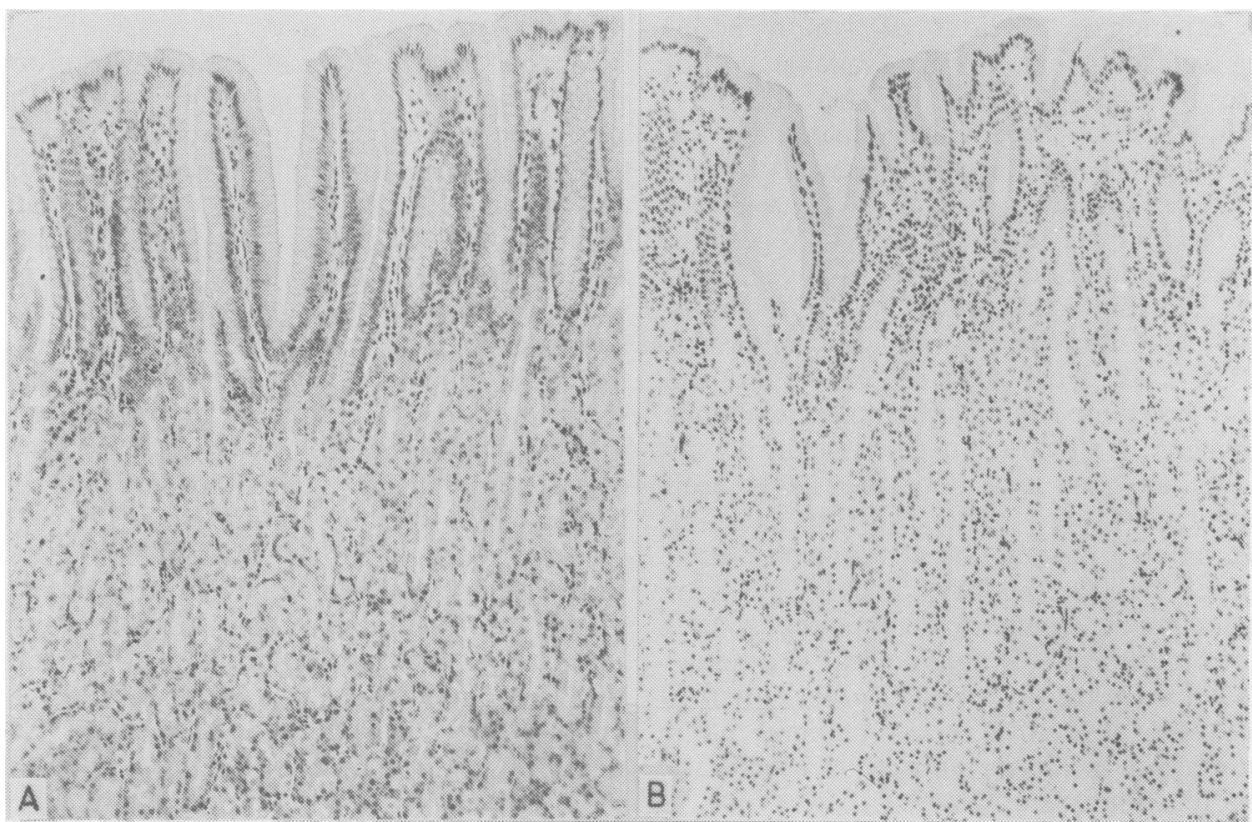

Fig. 1 Normal gastric body mucosa showing $(A)$ absence of inflammatory infiltrate, $(B)$ negative staining for secretory component. (A) haematoxylin and eosin, (B) immunoperoxidase, $\times 75$.

invasion. Dysplastic mucosa (Fig. 4) showed intense staining for secretory component and $\operatorname{IgA}$ often in the form of intracytoplasmic granules with heavy staining of the apical border of the glands. The intensity of the staining was greater than that found in inflamed or regenerative mucosa. Plasma cells staining for $\operatorname{IgA}$ were present in the intervening lamina propria in variable numbers. Dysplastic epithelium also stained positively for lysozyme but with uneven intensity, the strongly positive glands tending to be those furthest from the surface.

\section{CARCINOMA}

A total of 27 cases of carcinoma was studied including those tumours arising in the five cases of dysplasia described above. These were divided into tumours of so-called intestinal type and diffuse type following the classification proposed by Lauren.

The intestinal type of carcinoma, so-called because of its histological resemblance to large bowel carcinomas, was characterised by formation of distinct acini lined by malignant columnar cells. Differentiation varied markedly within the tumour and solid areas without gland formation were frequently present. Mucin secretion as shown by PAS staining consisted predominantly of accumulation within acini with individual cells staining poorly in well-differentiated areas and not at all in poorly-differentiated areas. All five tumours associated with foci of dysplasia were of intestinal type, as were 15 other cases.

The seven cases of diffuse gastric carcinoma consisted of infiltrates of loosely attached or individual cells with only occasional evidence of acinus formation. The individual cells were often very small, resembling macrophages. Larger cells contained a delicate, pale eosinophilic cytoplasm and sometimes had a clear signet ring configuration. Clear positive staining of individual cells for mucin was an important distinguishing feature of diffuse carcinoma.

It should be stressed that distinction between intestinal and diffuse types of carcinoma was not always clear cut. The seven cases classified as diffuse carcinomas consisted almost entirely of loosely arranged mucin-containing cells as described. Among the 20 cases of intestinal type carcinoma, however, small foci of diffuse carcinoma were often present and the mucin-containing cells in these foci demonstrated the immunohistochemical features of diffuse carcinoma as described below. Tumours consisting wholly of sheets of small undifferentiated cells which did not contain mucin were included in the intestinal category, although here, too, 


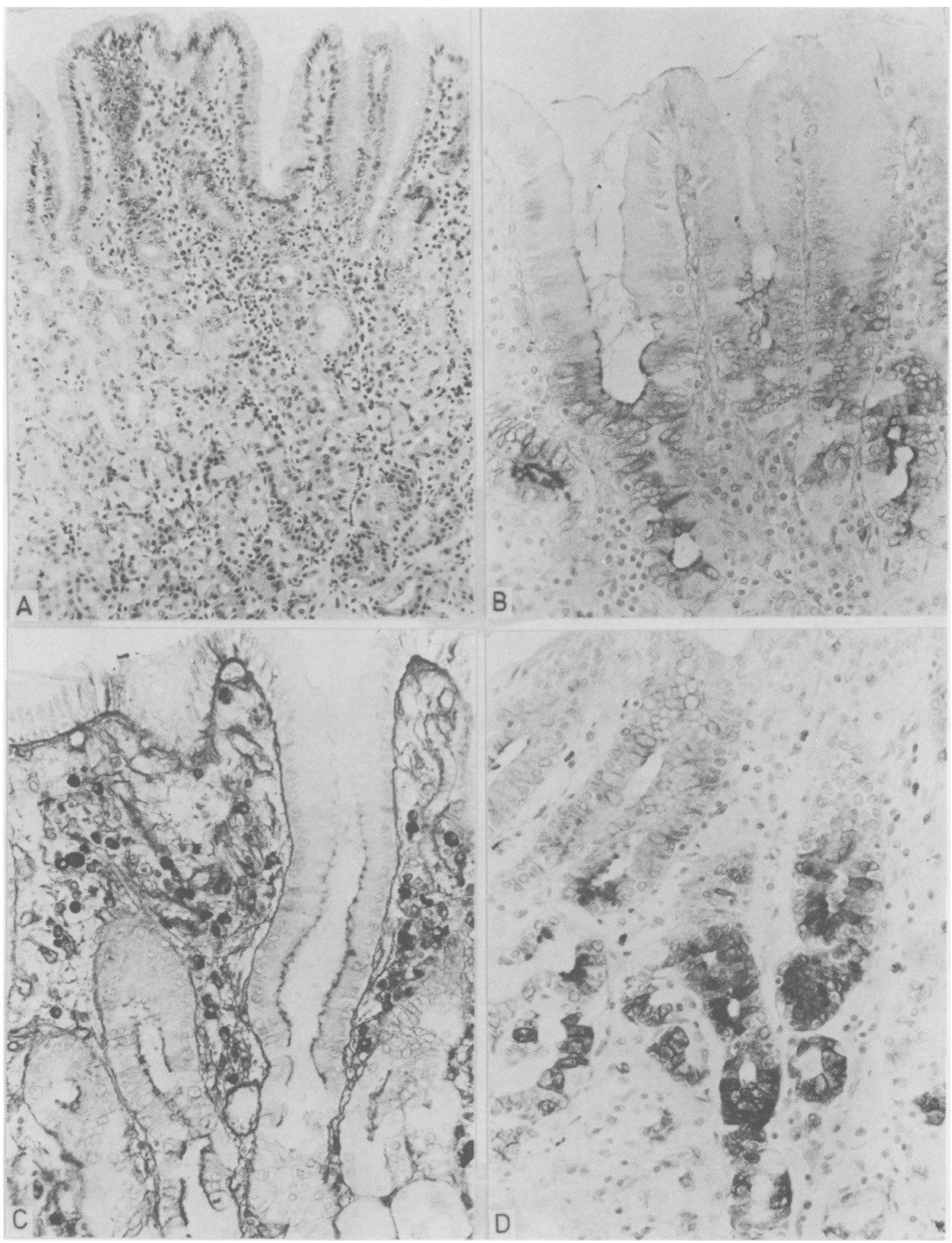

Fig. 2 Gastric body mucosa showing (A) chronic superficial gastritis, $(B)$ positive staining of mucous neck cells for secretory component, $(C)$ positive staining of plasma cells and mucous neck cells for $\operatorname{Ig} A,(D)$ positive staining of mucous neck cells for lysozyme. $(A)$ haematoxylin and eosin, $\times 100 ;(B)(C)$ and $(D)$ immunoperoxidase, $\times 175$. 


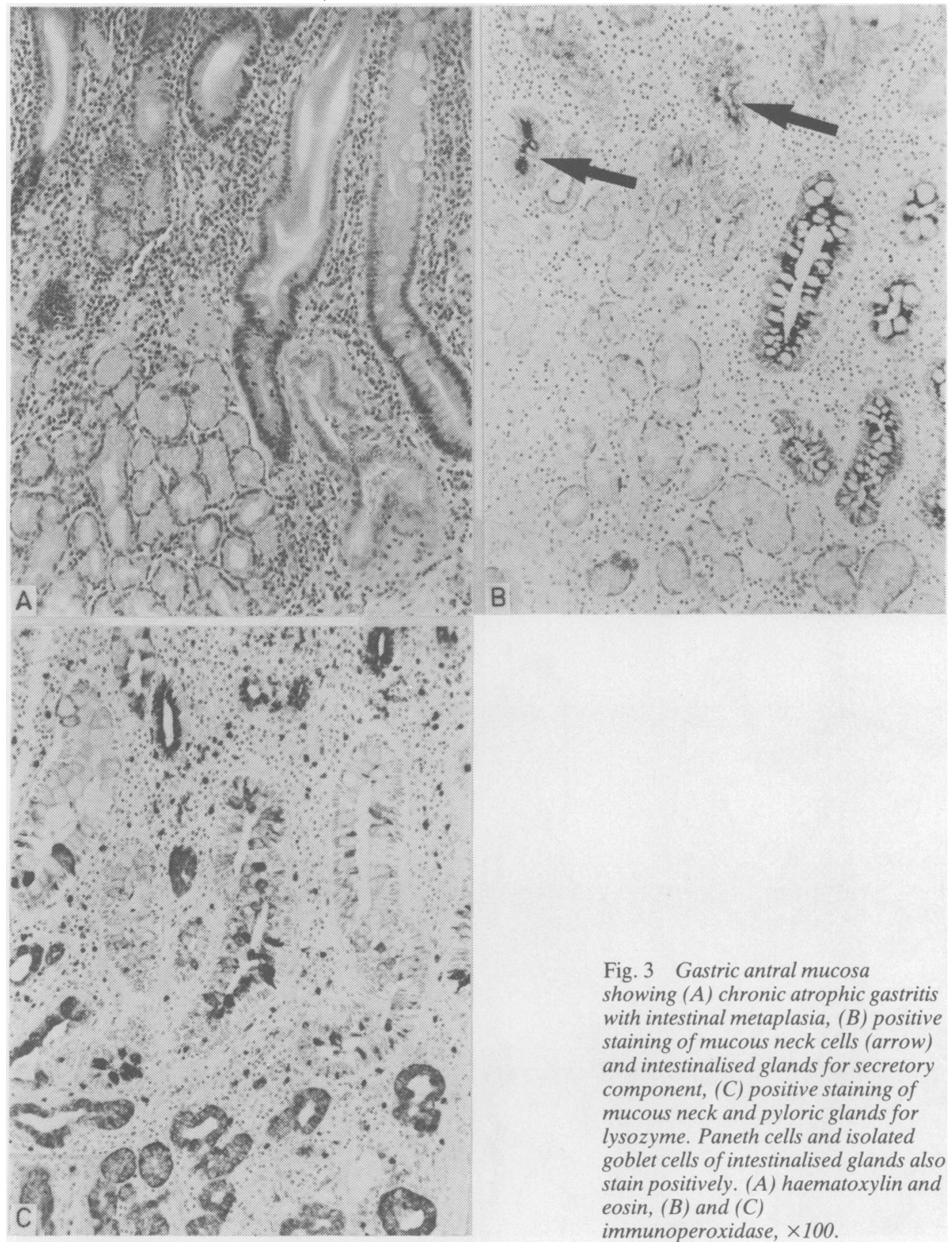



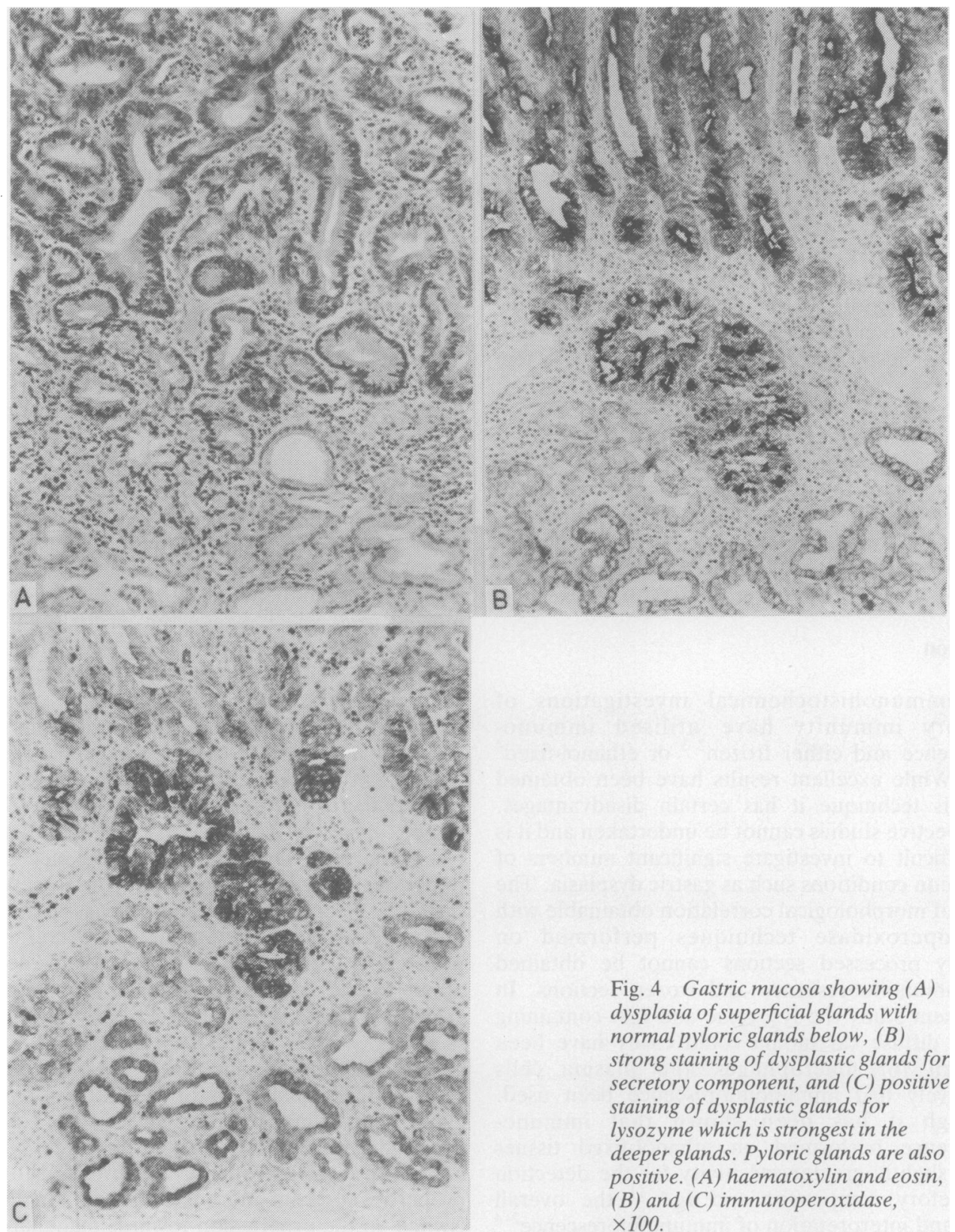
(1)

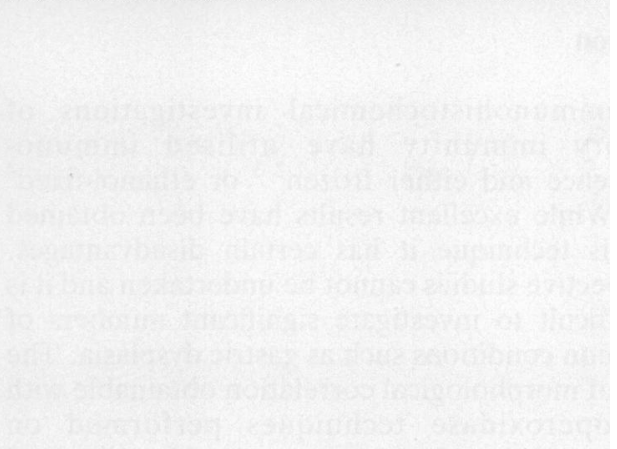

Fig. 4 Gastric mucosa showing $(A)$ dysplasia of superficial glands with normal pyloric glands below, $(B)$ strong staining of dysplastic glands for secretory component, and (C) positive staining of dysplastic glands for lysozyme which is strongest in the deeper glands. Pyloric glands are also positive. (A) haematoxylin and eosin, $(B)$ and $(C)$ immunoperoxidase, $\times 100$. 
occasional foci of mucin-containing cells were sometimes present.

The results of immunohistochemical staining were clearly different in the two types of carcinoma. Well-differentiated intestinal tumours stained positively for secretory component, $\operatorname{IgA}$, and lysozyme in a manner similar to dysplastic mucosa. As the tumours became less well-differentiated staining for all three antigens reduced in intensity or became negative (Fig. 5). Diffuse carcinomas stained strongly positive for secretory component, IgA, and lysozyme (Fig. 6). The positive staining for IgA was independent of the number of adjacent $\operatorname{IgA}$ containing plasma cells, which were usually very few in number.

\section{METASTATIC CARCINOMA IN LYMPH NODES}

The staining pattern of metastatic carcinoma reproduced that of the primary tumours described above (Fig. 7). The strong positive staining of metastatic diffuse carcinoma for all three antigens was again striking; only scattered $\operatorname{IgA}$ containing plasma cells were present in the lymph nodes.

\section{Discussion}

Most immunohistochemical investigations of secretory immunity have utilised immunofluorescence and either frozen ${ }^{23}$ or ethanol-fixed ${ }^{4}$ tissue. While excellent results have been obtained with this technique it has certain disadvantages. Retrospective studies cannot be undertaken and it is thus difficult to investigate significant numbers of uncommon conditions such as gastric dysplasia. The degree of morphological correlation obtainable with immunoperoxidase techniques performed on routinely processed sections cannot be obtained with immunofluorescence and frozen sections. In the present study the lysozyme and IgA-containing cells of diffuse carcinoma could easily have been mistaken for macrophages and plasma cells respectively had immunofluorescence been used. Although it has been shown that immunofluorescence performed on ethanol-fixed tissues affords slightly greater sensitivity for the detection of secretory component and IgA,${ }^{14}$ the overall results and interpretation of immunofluorescence ${ }^{2-4}$ and immunoperoxidase ${ }^{56}$ studies have been similar.

While numerous previous studies have documented the distribution of secretory component and $\operatorname{IgA}$ in the normal and diseased intestine, ${ }^{1-6} 14$ the stomach has received little attention. Using an immunofluorescence technique Ejekcam et al ${ }^{15}$ identified $\operatorname{IgA}$ in normal gastric glands of unspecified type and noted its presence in foci of intestinal metaplasia and in well- differentiated carcinomas as opposed to poorlydifferentiated tumours. Diffuse carcinomas were found to stain negatively for $\operatorname{IgA}$. It is noteworthy that in two separate immunoperoxidase studies on the distribution of lysozyme in normal tissues the stomach was described as negative in one ${ }^{16}$ while, in the later study, an occasional epithelial cell was reported as staining positively. ${ }^{15}$ In a specific investigation of the distribution of lysozyme in gastric mucosa, however, Reitamo et al, ${ }^{9}$ described the presence of lysozyme in antral and fundic glands. They noted that antral glands stained more consistently positive and that it was the mucous neck cells that stained positively in the fundus. These authors also described positive staining in the malignant cells of one case of gastric carcinoma.

Given the acid $\mathrm{pH}$ of the normal stomach, secretory immunity might not be considered to be a necessary function in this organ. This is borne out to some extent by the failure to demonstrate secretory component or $\operatorname{IgA}$ in uninflamed gastric body mucosa. It is probable that the small amount of secretory component and $\operatorname{IgA}$ detected in antral mucous neck cells is a reflection of the slight degree of inflammation present in the sections studied since no normal - that is, uninflamed - antral mucosa was available. With more florid evidence of gastritis mucous neck cells of both body and antral mucosa appeared to adapt by synthesising more secretory component and hence transporting IgA synthesised by the increased numbers of plasma cells. The strong positive staining of pyloric glands for lysozyme suggests that this substance has an important, but as yet undefined, role in gastric and intestinal secretions, presumably as part of the antibacterial defence system. Like secretory component, synthesis of lysozyme by mucous neck cells appears to be an adaptive response to inflammation.

While the acquisition of the ability to synthesise secretory component and lysozyme can be explained as an adaptive response in gastritis, there seems to be no logical explanation for dysplastic or malignant glands displaying these features. The fact that dysplastic mucosa and well-differentiated invasive malignancy share these acquired functional properties suggests, moreover, that the fundamental 'switch' in the progression towards malignancy may occur at the level of dysplasia. A similar suggestion has been made after a study of the secretory immune system in colorectal neoplasia where both dysplastic (adenomatous) mucosa and well-differentiated carcinomas synthesise secretory component and transport $\operatorname{IgA} .{ }^{6}$ The argument with respect to gastric mucosa carries rather more weight, however, as, unlike the colon, synthesis of lysozyme and secretory component reflects an alteration from 


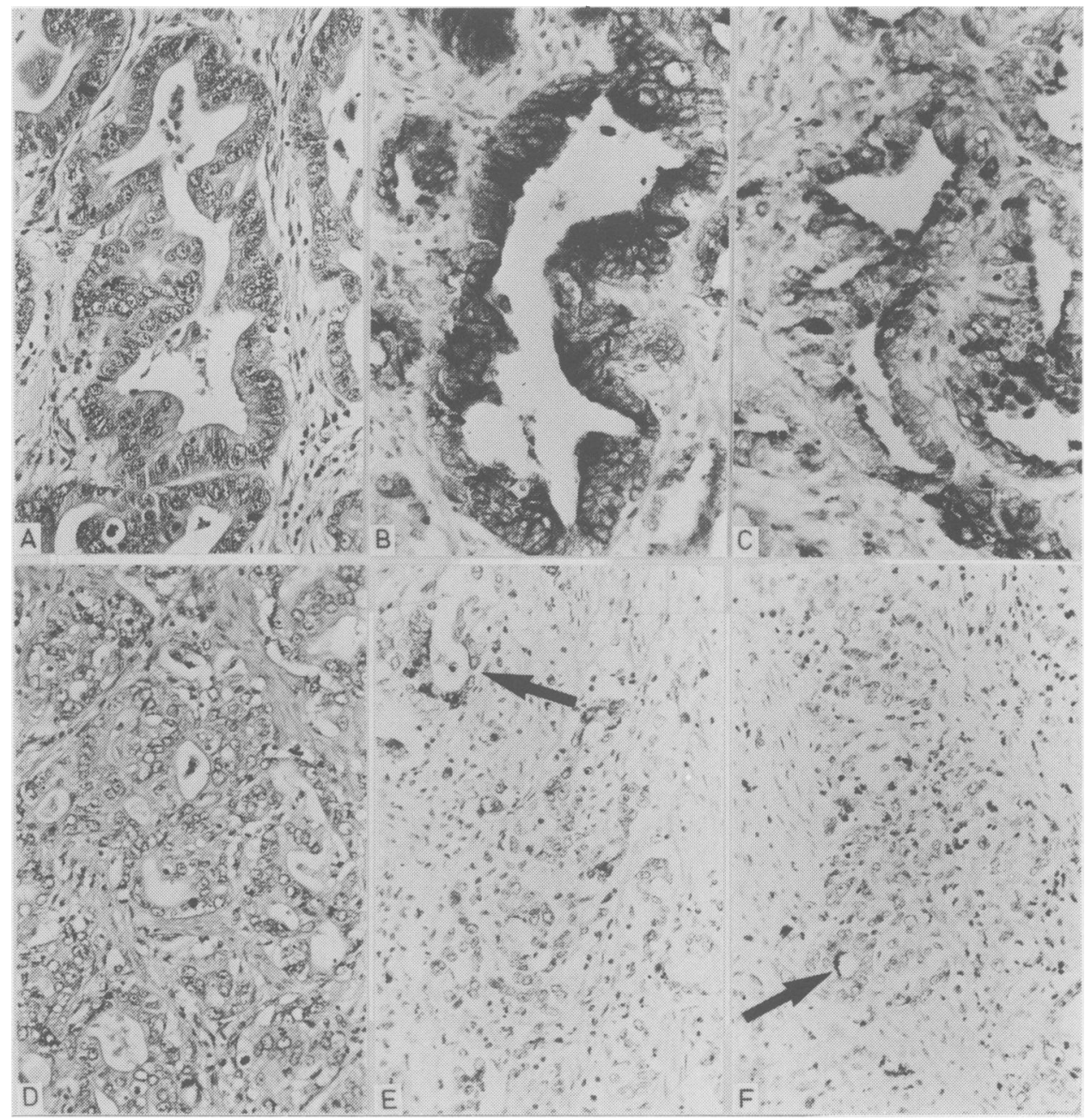

Fig. 5 Gastric adenocarcinoma of intestinal type showing $(A)$ well-differentiated area of tumour, $(B)$ strong staining for secretory component, and $(C)$ strong staining for lysozyme. (D) poorly-differentiated area of same tumour, $(E)$ staining only focally for secretory component (arrow), and $(F)$ lysozyme (arrow). $(A)$ and $(D)$ haematoxylin and eosin, $(B)(C)(E)$ and $(F)$ immunoperoxidase, $\times 200$.

normal mucosa. Positive staining for lysozyme in gastric dysplasia and well-differentiated carcinomas is in contrast to the analogous colonic lesions which stain variably and weakly for lysozyme (unpublished observations). The findings in diffuse carcinoma underline the suggestion made by Lauren ${ }^{13}$ that these tumours are not necessarily as "undifferentiated' as they appear, as not only mucin production but other normal functions of gastric epithelium are retained. These immunohistochemical studies serve to support Lauren's concepts of the two different types of carcinomas. As both gastric mucous neck cells and small intestinal crypt cells synthesise secretory component and lysozyme, however, they neither support nor contradict suggestions that intestinal type carcinomas arise in foci of intestinal metaplasia, while the diffuse tumours arise from unaltered foveolar cells.

It has been suggested previously that the $\operatorname{IgA}$ which can be demonstrated in secretory component synthesising malignant cells, in the absence of significant numbers of surrounding $\operatorname{IgA}$ secreting plasma cells, may be derived from circulating dimeric $\operatorname{IgA} .^{5}$ It is difficult to explain the entry of 


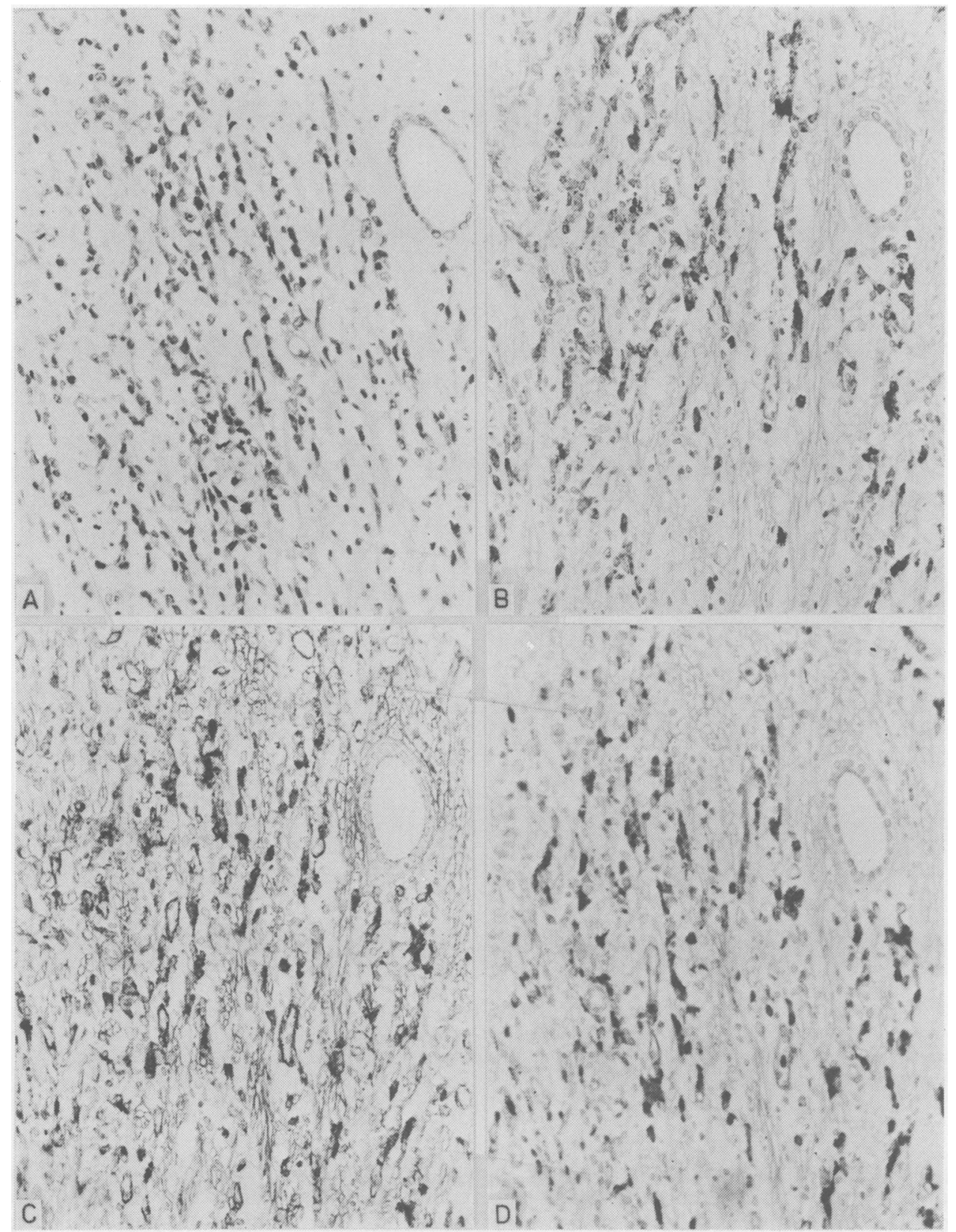

Fig. 6 Gastric adenocarcinoma of diffuse type showing $(A)$ invasion of submucosa by small mucin containing cells with benign gland at level of muscularis mucosae. The tumour cells stain positively for $(B)$ secretory component, $(C) I g A$, and (D) lysozyme. (A) PAS, (B) (C) and (D) immunoperoxidase, $\times 250$. 


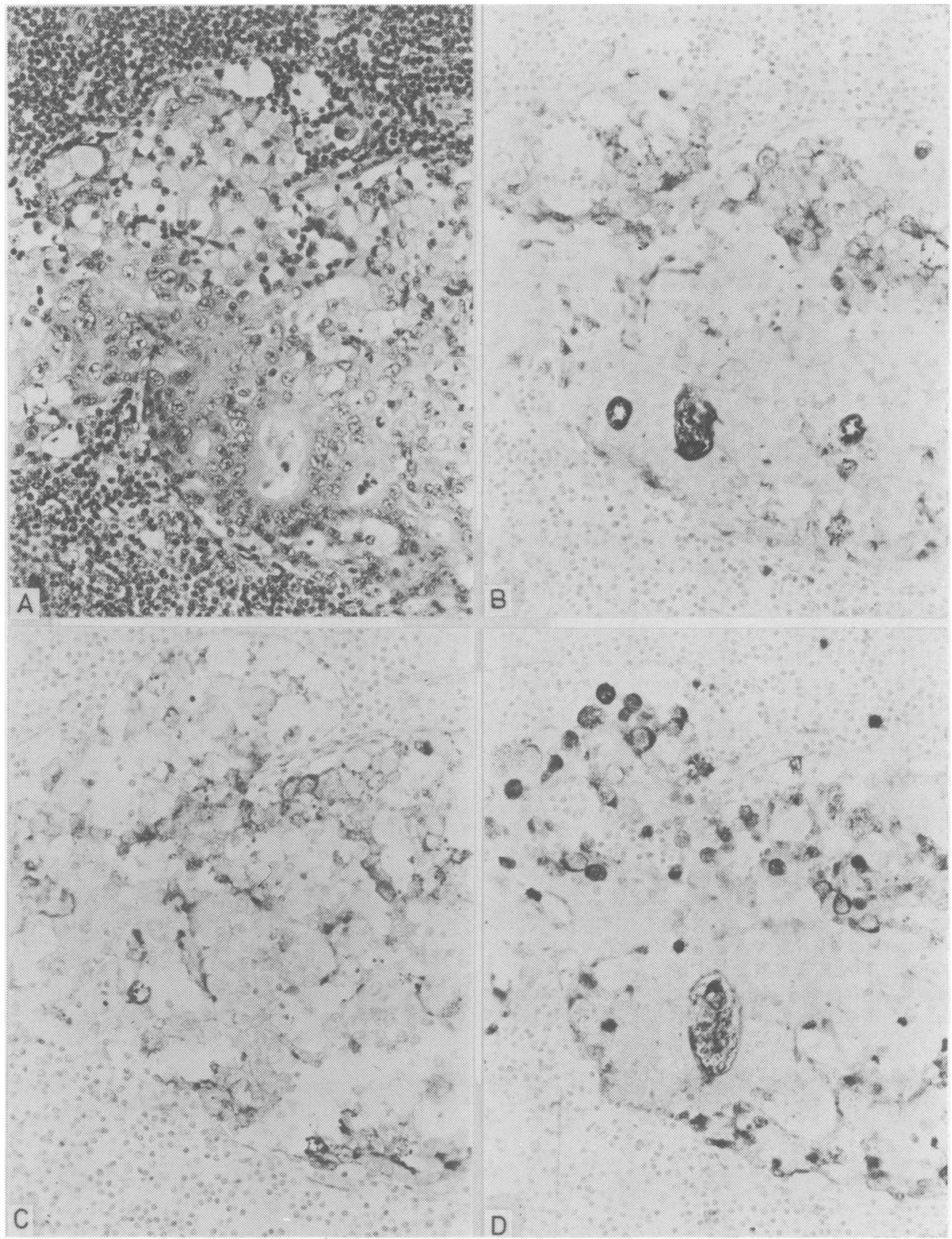

Fig. 7 Lymph node metastasis from a diffuse type gastric carcinoma showing $(A)$ signet ring cells and focal acinus formation. There is positive staining for $(B)$ secretory component, $(C) \operatorname{IgA}$, and $(D)$ lysozyme. $(A)$ haematoxylin and eosin, $(B)(C)$ and $(D)$ immunoperoxidase, $\times 200$. 
IgA into cells of diffuse gastric carcinoma deep in the muscularis of the stomach or in metastatic deposits in lymph nodes in any other way. Small amounts of IgM together with $\mathbf{J}$ chain can also be detected in these cells which, however, stain negatively for IgG (unpublished observations), further supporting their ability to bind and take up dimeric immunoglobuins. A similar mechanism has been suggested for the transport of circulating IgA into the bile by biliary epithelium ${ }^{18}$ and could be important in maintaining secretory immunity in mucosal tissues lacking an IgA secreting plasma cell compartment such as the uterine cervix.

\section{References}

1 Brandtzaeg P. Transport models for secretory IgA and secretory IgM. Clin Exp Immunol 1981; 44: 221-32.

2 Poger ME, Hirsch BR, Lamm ME. Synthesis of secretory component by colonic neoplasms. Am J Pathol 1976; 82: 327-34.

3 Weisz-Carrington P, Poger ME, Lamm ME. Secretory immunoglobulins in colonic neoplasms. Am J Pathol 1976; 85: 303-14.

4 Rognum TO, Brandtzaeg P, Örjasaeter H, Elgjo K, Hognestad J. Immunohistochemical study of secretory component, secretory $\operatorname{IgA}$ and carcinoembryonic antigen in large bowel carcinomas. Pathol Res Pract 1980; 170: $126-45$.

5 Isaacson P. Crypt cell carcinoma of the appendix (so-called adenocarcinoid tumor). Am J Surg Pathol 1981; 5: 213-24.

6 Isaacson P. An immunoperoxidase study of the secretory immunoglobulin system in colonic neoplasia. $J$ Clin Pathol 1982; 34: 14-25.

7 Montero C, Erlandsen SL. Immunocytochemical and histochemical studies on intestinal epithelial cells producing both lysozyme and mucosubstance. Anat Rec 1978; 190: 127-42.

8 Klockars M, Reitamo S. Tissue distribution of lysozyme in man. J Histochem Cytochem 1975; 23: 932-40.

9 Reitamo S, Reitamo JJ, Sipponen P, Klockars M. The distribution of lysozyme in human gastric mucosa in inflammatory and neoplastic disorders. Acta Pathol Microbiol Scand [A] 1979; 87: 451-6.

10 Mepham BL, Frater W, Mitchell BS. The use of proteolytic enzymes to improve immunoglobulin staining by the PAP technique. Histochem J 1979; 11: 345-57.

11 Isaacson P, Wright DH, Judd MA, Mepham BL. Primary gastrointestinal lymphomas: a classification of 66 cases. Cancer 1979; 43: 1805-19.

12 Morson BC, Sobin LH, Grundmann E, Johnson A, Nagayo T, Serck-Hansen A. Precancerous conditions and epithelial dysplasia in the stomach. J Clin Pathol 1980; 33: 711-21.

13 Lauren P. The two histological main types of gastric carcinoma: diffuse and so-called intestinal-type carcinoma. An attempt at a histo-clinical classification. Acta Pathol Microbiol Scand 1965; 64: 31-49.

14 Rognum TO, Brandtzaeg P, Ørjasaeter H, Fausa O. Immunohistochemistry of epithelial cell markers in normal and pathological colon mucosa. Comparison of results based on routine formalin and cold ethanol fixation methods. Histochemistry 1980; 67: 7-21.

15 Ejeckam GC, Huang SN, McCaughey WTE, Gold P. Immunohistopathologic study on carcinoembryonic antigen (CEA)-like material and immunoglobulin $\mathrm{A}$ in gastric malignancies. Cancer 1979; 44: 1606-14. .

16 Mason DY, Taylor CR. The distribution of muramidase (lysozyme) in human tissue. J Clin Pathol 1975; 28: 124-32.

17 Pinkus GS, Said JW. Profile of intracytoplasmic lysozyme in normal tissues, myeloproliferative disorders, hairy cell leukaemia, and other pathologic processes. An immunoperoxidase study of paraffin sections and smears. Am J Pathol 1977; 89: 351-62.

18 Nagura H, Smith PD, Nakane PK, Brown WR. IgA in human bile and liver. J Immunol 1981; 126: 587-95. 